\title{
CALCULATING EXTRUSION TEXTURES OF THE ALLOY Mn-Al-C
}

\author{
V. D. SOLOVEI and YU. N. LOGINOV \\ Institute of the Engineering Science, Urals State Technical University, \\ 91 Pervomaiskaja Str., 620219 Ekaterinburg GSP-207, Russia
}

(Received 8 April 1996)

The alloy Mn-Al-C (magnetic $\tau$-phase) has a face-centered tetragonal lattice* with the superstructure $L 1_{0}$ (Shangurov, Gornostyrev, Teitel et al., 1990). The tetragonal crystal axes $c$ (the directions of the easy magnetization) of the grain lattices of the polycrystal permanent magnet must be preferably oriented along the magnet axis. In the present paper the forming of the axial extrusion textures in the alloy $\mathrm{Mn}-\mathrm{Al}-\mathrm{C}$ is investigated theoretically. The texture inhomogeneity is taken into account by solving the boundary value problem of plasticity.

KEY WORDS: Extrusion texture, face-centered tetragonal lattice with the superstructure $L 1_{0}$, slip and twin systems, boundary value problem of plasticity.

\section{NOTATION}

$$
\begin{array}{ll}
\mathrm{x}, \mathrm{y}, \mathrm{z} & - \text { Cartesian coordinates** } \\
\rho, \varphi, \mathrm{z} & - \text { cylindrical coordinates } \\
\mathrm{r}, \vartheta, \varphi & - \text { spherical coordinates } \\
\omega_{i} & - \text { grain lattice rotation vector in the polycrystal } \\
\bar{\omega}_{i} & - \text { vector of the common rotation of the macroscopic particle in the } \\
& \text { polycrystal } \\
\omega_{i}^{a} & - \text { vector of the additional rotation of the grain lattice connected with } \\
& \text { the acting shear systems } * * * \text { and twin reorientation of the grain } \\
\varepsilon_{i j} & - \text { plastic strain tensor } \\
a_{i}^{(n)} & - \text { single vector of the shear direction for the n-th shear system } \\
b_{i}^{(n)} & - \text { single vector of the normal to the shear plane for the n-th shear } \\
\gamma^{(n)} & - \text { grain shear connected with the shear on the n-th shear system } \\
\varepsilon_{i j k} & - \text { Levi-Civita tensor }
\end{array}
$$

* Lattice means crystal lattice

** The Latin subscripts are referred to the Cartesian coordinates

*** The slip and twin systems hereinafter are called shear systems 


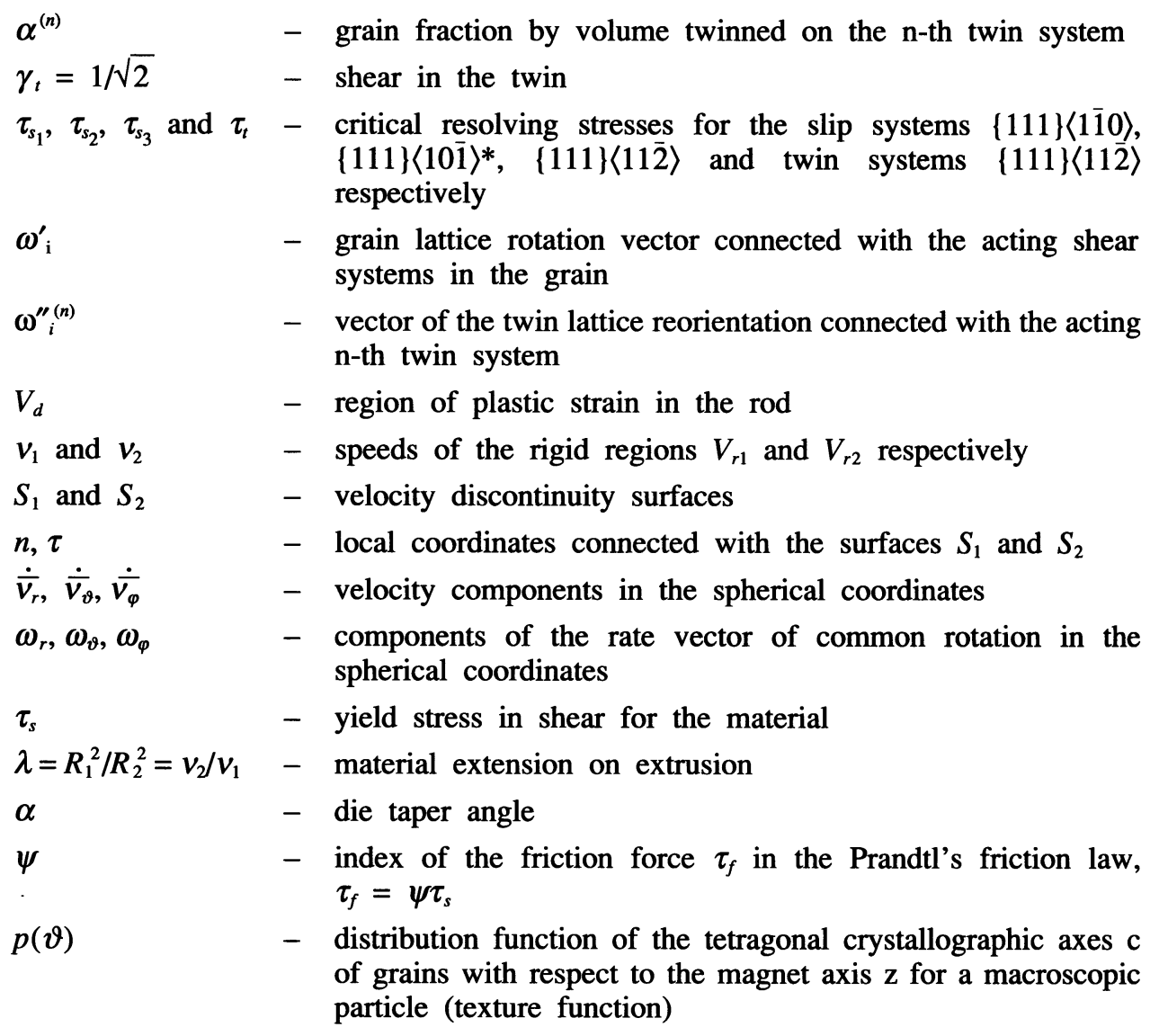

\section{INTRODUCTION}

The calculation of the deformation textures comes to calculating the lattice rotation vector for the arbitrarily oriented grain, which can be represented by the formula (Taylor, 1938; Bunge, 1970; Houtte and Aernoudt, 1975)

$$
\omega_{i}=\bar{\omega}_{i}+\omega_{i}^{a}
$$

The vector of the common rotation $\bar{\omega}_{i}$ in the case of inhomogeneous strain can be found from the boundary value problem of macroscopic plasticity. The vector of the additional rotation of the grain lattice $\omega_{i}^{a}$ is determined by the acting shear systems and the twin reorientation of the grain lattice.

* The first two indexes and the third index in the designation of the crystallographical direction are physically inequivalent for the tetragonal lattice with the superstructure $L 1_{0}$ (stratiform arrangement of the $\mathrm{Mn}$ and $\mathrm{Al}$ atoms in the lattice). 


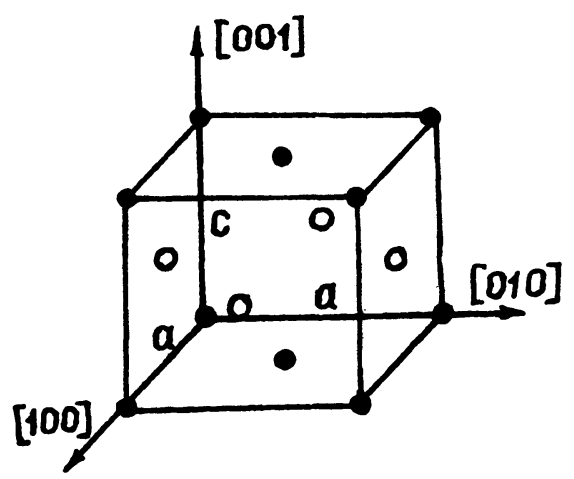

Figure 1 A primary crystal cell of the alloy $\mathrm{Mn}-\mathrm{Al}-\mathrm{C}$ in the $\tau$-phase $(-\mathrm{Mn}$ atoms; $\bigcirc-\mathrm{Al}$ atoms; $\mathrm{a}=3.91 \AA ; \mathrm{c}=3.63 \AA)$.

\section{CALCULATING ADDITIONAL ROTATIONS OF GRAIN LATTICES}

The primary crystal cell of the $\tau$-phase of the alloy $\mathrm{Mn}-\mathrm{Al}-\mathrm{C}$ is shown in Figure 1. The plastic strain of this crystal occurs with shears on the slip systems $\{111\}\langle 110\rangle$, $\{111\}\langle 10 \overline{1}\rangle,\{111\}\langle 11 \overline{2}\rangle$ and twin systems $\{111\}\langle 11 \overline{2}\rangle$ (Shangurov, Gornostyrev, Teitel et al., 1990).

The additional rotations of grain lattices are calculated as in the paper (Chin, Hosford and Mendorf, 1969).

According to the Taylor approximation (Taylor, 1938), it is assumed that the strain of the arbitrary grain is equal to the macroscopic strain.

Virtual sets of five shear systems and corresponding grain shears must provide the given strain

$$
\varepsilon_{i j}=\frac{1}{2} \sum_{n=1}^{5}\left(a_{i}^{(n)} b_{j}^{(n)}+a_{j}^{(n)} b_{i}^{(n)}\right) \gamma^{(n)}
$$

where summation spreads over all the shear systems of the virtual set.

Connected with the shear on the $\mathbf{k}$-th twin system, the grain shear $\gamma^{(k)}$ determines the fraction by volume of the grain region twinned on this twin system $\left(\alpha^{(k)}\right)$ in the following way:

$$
\gamma^{(k)}=\alpha^{(k)} \gamma_{t}
$$

The acting sets of five shear systems and the corresponding grain shears are found among the virtual ones from the minimum condition of the work of the critical resolved stresses on the shear systems, which can be written as

$$
\sum_{n=1}^{5} \tau^{(n)} \gamma^{(n)} \rightarrow \min
$$

where $\tau^{(n)}=\tau_{s_{1}}, \tau_{s_{2}}, \tau_{s_{3}}$ and $\tau_{t}$ for the slip systems $\{111\}\langle 1 \overline{1} 0\rangle,\{111\}\langle 10 \overline{1}\rangle$, $\{111\}\langle 11 \overline{2}\rangle$ and twin systems $\{111\}\langle 11 \overline{2}\rangle$ respectively.

If several acting sets of five shear systems and corresponding grain shears exist, it is physically justified to take into account all these sets and shears (Bunge and Fuchs, 1969). 
The vector of grain lattice rotation $\omega_{i}^{\prime}$ determined by the acting set of five shear systems and corresponding grain shears is described by the following formula:

$$
\omega_{i}^{\prime}=-\frac{1}{2} \varepsilon_{i j k} \sum_{n=1}^{5}\left(b_{j}^{(n)} a_{k}^{(n)}-b_{k}^{(n)} a_{j}^{(n)}\right) \gamma^{(n)}
$$

If several acting sets of five shear systems and the corresponding grain shears exist, this rotation is found as the mean rotation for all these sets and shears.

The vector of twin reorientation $\omega_{i}^{\prime \prime(k)}$ of the grain lattice connected with the twinning on the k-th twin system is written in the following form:

$$
\omega_{i}^{\prime \prime(k)}=\omega \varepsilon_{i j p} a_{j}^{(k)} b_{p}^{(k)}
$$

where $\omega=1.23096$.

In the grain region where slipping alone takes place, the additional rotation of the lattice $\omega_{i}^{a}$ is determined by the formula

$$
\omega_{i}^{a}=\omega_{i}^{\prime}
$$

whereas in the grain region where twinning on the k-th twin system takes place, this rotation is represented by the formula

$$
\omega_{i}^{a}=\omega_{i}^{\prime}+\omega_{i}^{\prime \prime(k)}
$$

In the present paper we take into account only the slip systems $\{111\}\langle 1 \overline{1} 0\rangle$, $\{111\}\langle 10 \overline{1}\rangle$ and the twin systems $\{111\}\langle 11 \overline{2}\rangle$. We also assume the equalities for the lattice parameters $a=c$ and for the critical resolved stresses $\tau_{s_{1}}=\tau_{s_{2}}$. The critical resolved stress $\tau_{t}$ is determined with help of the following reasons. On the one hand, the twinning makes a substantial contribution to the deformation of the alloy Mn-Al-C (Shangurov, Gornostyrev, Teitel et al., 1990), therefore this stress must be small enough. On the other hand, the calculations show that, for the uniaxial compression of face-centered cubic crystals, the only stable equiponderant orientation $\langle 110\rangle$ exists on condition that $\tau_{t} \geq \tau_{s_{1}} / \sqrt{3}$. Taking into account these reasons, we assume that $\tau_{t}=0.6 \tau_{s 1}$.

\section{YIELD KINEMATICS OF THE ROD UNDER EXTRUSION}

We use the solution of the boundary value problem of Mises's ideal plasticity (Mises, 1928) for the rod extrusion process (Figure 2). The solution was obtained in the works of Stepanenko (1969) and Kolmogorov et al. (1993).

The radii $r_{1}$ and $r_{2}$ of the boundary surfaces $S_{1}$ and $S_{2}$ respectively for the deformed region $V_{d}$, as well as the velocity components $v_{r}, v_{\vartheta}, v_{\varphi}$ and the rate vector components of common rotation $\dot{\bar{\omega}}_{r}, \dot{\bar{\omega}}_{\vartheta}, \dot{\bar{\omega}}_{\varphi}$ for the particles in this region, are determined by the following relations:

$$
r_{1}=R_{1}(h \operatorname{Cos} \vartheta+f), r_{2}=r_{1} / \sqrt{\lambda}
$$




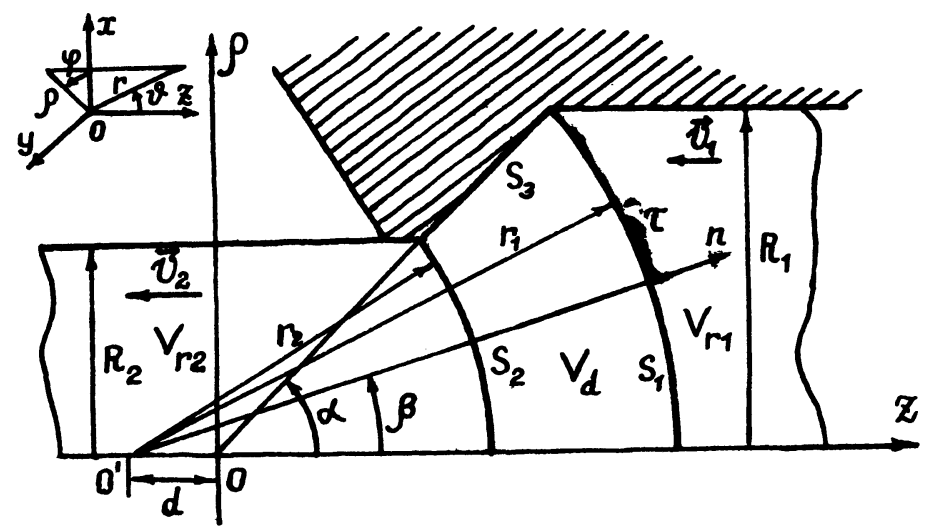

Figure 2 The scheme of rod extrusion through a conic die.

$$
\begin{aligned}
& v_{r}=v_{1} r_{1}^{2}\left(\operatorname{Cos} \vartheta-h \operatorname{Sin}^{2} \vartheta / f\right) / r^{2}, v_{\vartheta}=0 ; v_{\varphi}=0 ; \\
& \dot{\bar{\omega}}_{r}=0, \dot{\bar{\omega}}_{\vartheta}=0, \dot{\bar{\omega}}_{\varphi}=v_{1} r_{1}^{2}(\operatorname{Sin} \vartheta+h \operatorname{Sin} 2 \vartheta / f) / 2 r^{3},
\end{aligned}
$$

where $h=d / R_{1}^{*}, f=\left[1+(\operatorname{Ctg} \alpha-h)^{2}-h^{2} \operatorname{Sin}^{2} \vartheta\right]^{\frac{1}{2}}$.

The motion trajectories of the particles are easy found by the integration of velocity with respect to time.

The strain tensor components and the vector components of common rotation in the region $V_{d}$ are defined by the following expressions:

$$
\begin{aligned}
& \varepsilon_{r r}=\ln r_{1}^{2} / r^{2}, \varepsilon_{\vartheta \vartheta}=\varepsilon_{\varphi \varphi}=-\ln r_{1} / r, \\
& \varepsilon_{r \vartheta}=-\bar{\omega}_{\varphi}=\frac{1}{2} \frac{f \operatorname{Sin} \vartheta+h \operatorname{Sin} 2 \vartheta}{f \operatorname{Cos} \vartheta-h \operatorname{Cos}^{2} \vartheta} \ln r_{1} / r .
\end{aligned}
$$

The velocity components on the velocity discontinuity surfaces $S_{1}$ and $S_{2}$ in the local coordinates $n, \tau$ are written as

and

$$
v_{\tau 1}^{-}=v_{1} \operatorname{Sin} \beta, \quad v_{\tau 1}^{+}=\left.v_{\mathrm{r}}\right|_{r=\mathrm{r} 1} \operatorname{Sin}(\beta-\vartheta), \quad v_{n 1}=-v_{1} \operatorname{Cos} \beta
$$

$$
v_{\tau 2}^{-}=\left.v_{\mathrm{r}}\right|_{r=\mathrm{r} 2} \operatorname{Sin}(\beta-\vartheta), \quad v_{\tau 2}^{+}=v_{1} \lambda \operatorname{Sin} \beta, \quad v_{n 2}=-v_{1} \lambda \operatorname{Cos} \beta
$$

respectively. Here minus (plus) refers to the position of the particle in front of (behind) the velocity discontinuity surface.

On the velocity discontinuity surfaces $S_{1}$ and $S_{2}$, the strains and the common rotations of the particles have equal moduli and opposite signs, therefore they are not taken into account in the calculations.

*Here $h$ is an undetermined parameter. 
The parameter $h$ is determined from the minimum condition for the complete power $N$ described by the following relations:

$$
\begin{aligned}
& N=N_{V}+N_{1}+N_{2}+N_{3}, \\
& N_{V}=2 \sqrt{2} \pi \tau_{s} \int_{r_{2}}^{r_{1}} \int_{0}^{\alpha}\left[\left(\partial v_{r} / \partial r\right)^{2}+2\left(v_{\mathrm{r}} / r\right)^{2}+\left(\partial v_{r} / \partial \vartheta\right)^{2} / 2 r\right]^{1 / 2} r^{2} \operatorname{Sin} \vartheta d \vartheta d r \\
& N_{\delta}=2 \pi \tau_{s} \int_{0}^{\alpha}\left|v_{\tau \delta}^{-}-v_{\tau \delta}^{+}\right|_{r=r_{s}} r_{\delta}^{2} \operatorname{Sin} \vartheta d \vartheta \\
& N_{3}=2 \pi \psi \tau_{s} \operatorname{Sin} \alpha \int_{r_{2}}^{r_{1}}\left|v_{r}\right|_{\vartheta=\alpha} d r
\end{aligned}
$$

where $N_{V}$ is the deformation power in the volume $V d ; N_{\delta}$ is the power of the cutting forces on the velocity discontinuity surface $S_{\delta}(\delta=1,2) ; N_{3}$ is the power of the friction forces on the surface of the contact between the alloy and the die.

\section{CALCULATED EXTRUSION TEXTURES OF THE ALLOY Mn-Al-C}

In the space of the Eulerian angles*, the distribution function of the grain lattice orientations in the polycrystal $g\left(\varphi_{1}, \vartheta, \varphi_{2}\right)$ is described discretely on the cells having dimensions 45, 10 and 45 degrees for the angles $\varphi_{1}, \vartheta$ and $\varphi_{2}$ respectively. In the initial state, the rod has a random texture.

The strain of the macroscopic particles and the grain lattice rotations in these particles are calculated along the particle motion trajectories by the formulas from the previous paragraphs.

In order to decrease calculation errors connected with problem nonlinearity, we split the motion trajectories of the particles into 25 steps and sum the abovementioned strains and rotations on the steps.

Upon the deformation, the texture function $f(\vartheta)$ of the particle is obtained from the corresponding discrete distribution function of the grain lattice orientations $g\left(\varphi_{1}, \vartheta\right.$, $\varphi_{2}$ ) by means of the summation in the angles $\varphi_{1}$ and $\varphi_{2}$ followed by the approximation by means of the smooth function.

The calculation results for the texture of the alloy Mn-Al-C upon extrusion are presented in Figure 3 and Table 1. The textures are calculated for the macroscopic particles in the center and on the surface of the deformed rod.

The calculations show that, in the range $0.25 \leq \psi \leq 0.75$, the texture of the deformed rod depends on the friction force index only slightly, therefore this dependence is not presented here, and all the texture functions are presented for $\psi=0.5$.

From the Figure $3 \mathrm{a}$ it is seen that the texture perfection degree** increases as the extension value increases. As the extension reaches the value $\lambda=5.0$, the increase in the texture perfection degree slows down. A similar behaviour of the magnetic

\footnotetext{
*Eulerian angles $\varphi_{1}, \vartheta, \varphi_{2}$ determine the orientation of the principal crystallographic directions [100], [010] and [001] of the lattice in the coordinates $x, y, z$..

**The increase in the texture perfection degree denotes the increase in the sharpness and homogeneity of the axial texture [001].
} 

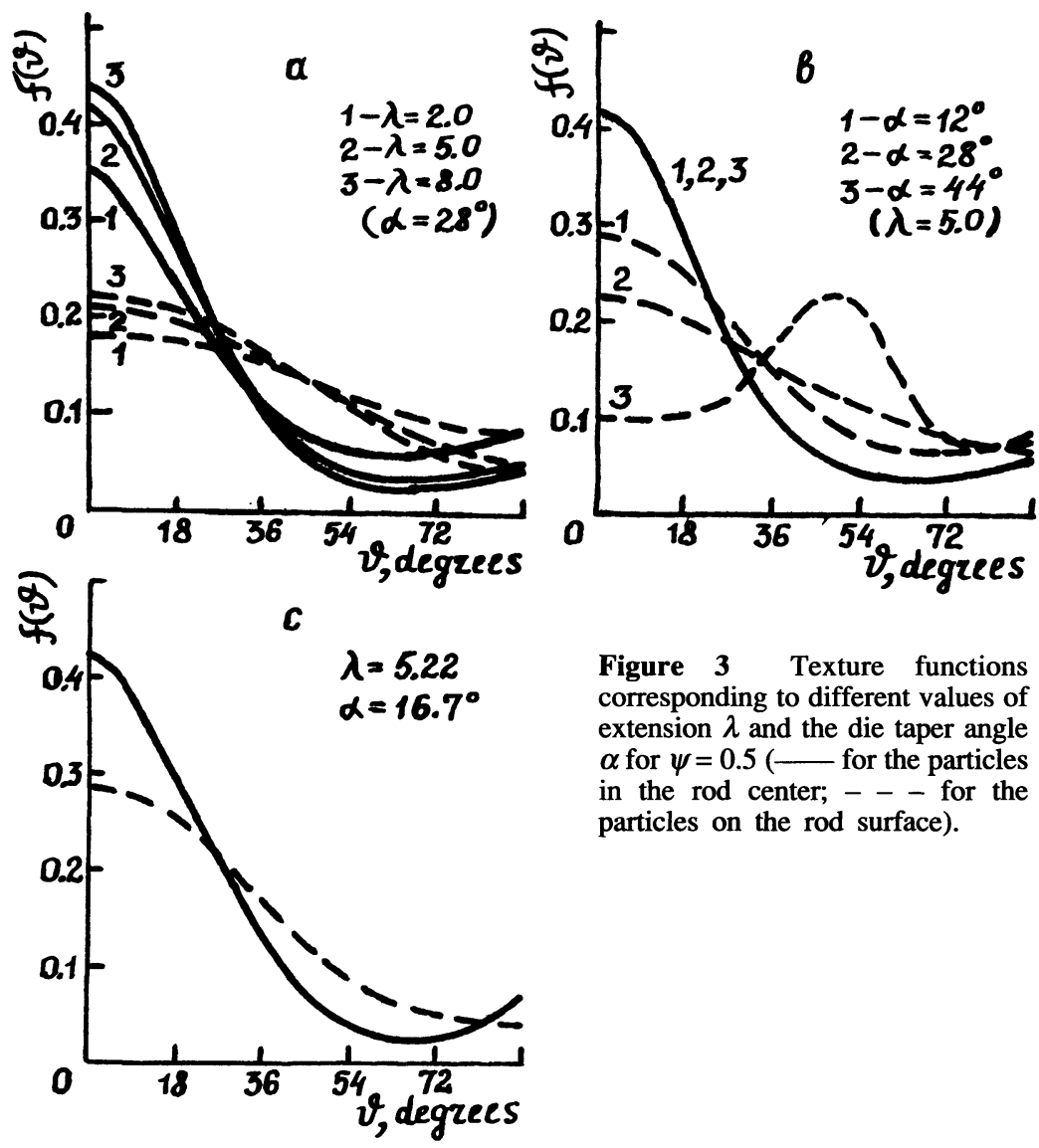

Figure 3 Texture functions corresponding to different values of extension $\lambda$ and the die taper angle $\alpha$ for $\psi=0.5$ ( - for the particles in the rod center; - - - for the particles on the rod surface).

Table 1 The critical die taper angle $\alpha_{c}$ (in degrees) for different values of extension $\lambda$ and the friction force index $\psi$.

\begin{tabular}{|l|l|l|l|}
\hline$\lambda$ & 0.25 & 0.50 & 0.75 \\
\hline 2.0 & & & \\
\hline 5.0 & 57.2 & 53.9 & 53.1 \\
8.0 & 54.6 & 49.0 & 46.8 \\
\hline
\end{tabular}

properties of the alloy $\mathrm{Mn}-\mathrm{Al}-\mathrm{C}$ with the extension increase was found experimentally in the work (USSR inventor's certificate, 1976). Taking into account this fact, as well as the great increase rate of the extrusion force with the increase in the extension for $\lambda>0.5$, the authors (USSR inventor's certificate, 1976) offered the following optimal extension value for the alloy Mn-Al-C: $\lambda_{o p}=5.22$. 
The dependence of the texture perfection degree on the die taper angle $\alpha$ has a complex character (see Figure 3,b).

The investigations of the strain state of the extruded rod permit to obtain a restriction for the optimal die taper angle $\alpha_{o p}$. We define the critical die taper angle $\alpha_{c}$ for which the strain tensor component $\varepsilon_{z z}$ on the surface of the deformed rod is equal to zero. This angle depends on the extension $\lambda$ and the friction force index $\psi$ (see Table 1). It is evident that the following restriction exists for the optimal die taper angle: $\alpha_{o p}<\alpha_{c}$.

In the work (Japan patent, 1974), the optimal die taper angle was found experimentally. When the die taper angle $\alpha$ increases, the dimensions of the extrusion tool decrease. Yet the turbulent motion of the alloy and the stagnation in the region of contact between the alloy and the tool emerging for great angles $\alpha$ decrease the texture perfection degree. The optimum die taper angle found in (Japan patent, 1974) is $\alpha_{o p}=16.7$ degrees.

The texture functions for the optimum values of extrusion parameters $(\lambda=5.22$, $\alpha=16.7^{\circ}, \psi=0.5$ ) are shown in Figure $3 c$. This texture is the best one among all the textures described in the present article.

\section{CONCLUSION}

The deformation texture theory taking into account crystallographic slipping and twinning is applied to the extrusion texture calculation for the alloy $\mathrm{Mn}$-Al-C having a face-centered tetragonal crystal lattice with the superstructure $L 1_{0}$. The texture inhomogeneity in the section of the rod made of the alloy Mn-Al-C is investigated by means of the boundary value problem for continuum mechanics. The calculation results agree with the known experimental data. It is shown that texture inhomogeneity is an essential optimization factor for the texture state in the process under study.

\section{References}

Bunge, H. J. and Fuchs, R. (1969). On the Orientation Changes during Plastic Deformation of Individual Crystallites in Polycrystalline Aggregates. Physica status solidi, 32, 169-177.

Bunge, H. J. (1970). Some Application of the Taylor Theory of Polycrystal Plasticity. Kristall und Technik, 5, 145-175.

Chin, G. Y., Hosford, W. F and Mendorf, D. R. (1969). Accommodation of constrained deformation in f.c.c. metals by slip and twinning. Proceeding of the Royal Society, A, 309, 433-456.

Patent of Japan N 54-42651. The production method of the magnets from the alloy Mn-Al-C. 11.07.74. MKI C22F1/16, C22C22/00 H01F 1/04.

USSR inventor's certificate N 820665. The production method of the magnetic alloy. 12.06.76. MKI C21D 8/12, BI, N 13, 1981.

Houtte, P. and Aernoudt, E. (1975) Solution of the Generalized Taylor Theory of Plastic Flow. Part 2. Zeitschrift fur Metallkunde, 66, 202-209.

Kolmogorov, V. L., Parshakov, S. I., Burkin, S. P., Loginov, Yu. N., Berezovsky, B. N. and Konovalov, A.V. et al. (1993). Solving the problems of metal forming on a micro-computer. M.: Metallurgiya, 320 P. (in Russian).

Mises, R. (1928). Mechanik der plastischen Formanderung von Kristallen. Zeitschrift fur angewandte Mathematik und Mechanik, 8, 161-185.

Shangurov, A.V., Gornostyrev, Yu. N., Teitel, E. I., Gundyrev, V. M., Yermakov, A. E. and Solovei, V. D. (1990). Texture forming during cold deformation of the alloy Mn-Al-C ordered similarly to the type $L 1_{0}$. Fizika metallov i metallovedenie, 6, 95-101 (in Russian).

Stepanenko, V. I. (1969). Investigation of forces and deformations in the processes of extrusion, drawing and die forging. Dissertatsia na soiskanie uchenoi stepeni kandidata tekhnicheskikh nauk, 51-60. Sverdlovsk: UPI (in Russian).

Taylor, G. I. (1938). Plastic strain in metals. Journal of the Institute of Metals, 62, 307-324. 